\title{
Finite-State Markov Modeling of Correlated Rician-Fading Channels
}

\author{
Cecilio Pimentel, Member, IEEE, Tiago H. Falk, Student Member, IEEE, and Luciano Lisbôa
}

\begin{abstract}
Stochastic properties of the binary channel that describe the successes and failures of the transmission of a modulated signal over a time-correlated flat-fading channel are considered for investigation. This analysis is employed to develop $K$ th-order Markov models for such a burst channel. The order of the Markov model that generates accurate analytical models is estimated for a broad range of fading environments. The parameterization and accuracy of an important class of hidden Markov models, known as the Gilbert-Elliott channel (GEC), are also investigated. Fading rates are identified in which the $K$ th-order Markov model and the GEC model approximate the fading channel with similar accuracy. The latter model is useful for approximating slowly fading processes, since it provides a more compact parameterization.
\end{abstract}

Index Terms-Flat fading, Gilbert-Elliott channels (GECs), Markov processes, parameter estimation, statistics of burst channels.

\section{INTRODUCTION}

$\mathbf{I}$ $\mathrm{N}$ A typical mobile communication channel, the transmitted signal undergoes attenuation and distortion caused by multipath propagation and shadowing. The nonfrequency-selective (flat) fading channel imposes multiplicative narrow-band complex Gaussian noise (referred to as the fading process) on the transmitted signal. As a consequence, abrupt changes in the mean received signal level may occur and the autocorrelation function $(\mathrm{ACF})$ of the fading process may lead to the occurrence of a burst of bit errors. The analytical analysis of such a bursty communication system involves the calculation of the multivariate probability density function (pdf) of the correlated fading process [1]-[4].

Finite state channel (FSC) models have been widely accepted as an effective approach to characterize the correlation structure of the fading process [5]-[20]. An FSC is described by a deterministic or probabilistic function of a first-order Markov chain, where each state may be associated with a particular channel quality. The strategy adopted by many researchers to

Manuscript received May 2, 2003; revised December 23, 2003 and March 2,2004 . This work was supported in part by the Brazilian National Council for Scientific and Technological Development (CNPq) under Grant 302402/2002-0.

C. Pimentel is with the Department of Electronics and Systems, Communications Research Group (CODEC), Federal University of Pernambuco, Recife, PE 52051-200, Brazil.

T. H. Falk was with the Department of Electronics and Systems, Federal University of Pernambuco, Recife, PE 52051-200, Brazil. He is now with the Department of Electrical and Computer Engineering, Queen's University, Kingston, ON K71 3N6, Canada.

L. Lisbôa was with the Department of Electronics and Systems, Federal University of Pernambuco, Recife, PE 52051-200, Brazil. He is now with CHESF, Recife, PE 52051-200, Brazil.

Digital Object Identifier 10.1109/TVT.2004.832413 design FSC models for fading channels consists of representing each state of a first-order Markov chain by a nonoverlapping interval of the received instantaneous signal-to-noise ratio (SNR)[6]-[16]. Criteria for partitioning the SNR have been discussed in [6], [11] and [16]. The modulation and demodulation schemes are incorporated into the model through the crossover probability of the binary symmetric channel associated with each fading state. An information theoretic metric has been proposed in [7] to validate the first-order model. The limitations of this criterion and the applicability of the first-order assumption have been discussed in [16]. Other model structures have also been proposed to represent the quantized SNR, including higher order Markov models [17], general hidden Markov models [20], Gilbert-Elliott channels (GECs) [18], and two-dimensional (2-D) models [19].

This paper concerns the development of FSC models for a discrete communication system composed by a frequency-shift-keying (FSK) modulator, a time-correlated Rician flat-fading channel, and a hard quantized noncoherent demodulator. The FSC model describes the successes and failures of the symbol transmitted over the fading channel, which is represented mathematically as a binary error sequence. The majority of models previously reported in the literature to characterize the symbol-by-symbol dynamics of the error sequence is based on a particular class of hidden Markov models called the simplified Fritchman model [4], [5]. Models based on a finite queue have been proposed recently [28].

The main contribution of this paper is to develop a methodology to find accurate $K$ th-order Markov models for such a bursty communication system. The order of the Markov process gives useful information on the memory of the error process or on how far the successive transmissions over the channel are temporarily related. We have applied several statistics to judge model accuracy and to estimate its order. The effect of the SNR, fading rate, and Rician factor on the accuracy of the proposed models is analyzed. As the fading rate becomes slower, the model may grow to inconvenient sizes. Our second contribution is to identify the system parameters in which the well-known two-state GEC model satisfactorily approximates the discrete communication channel. The results presented here allow us to accurately study coding performance on correlated fading channels using the analytical techniques developed in [18]-[27] to analyze burst channels represented as specific FSC models.

This paper is organized into six sections. Section II describes a communication system with flat fading and some properties of FSC models. Section III presents a methodology to estimate the parameters of two classes of FSC models: the $K$ th-order 
Markov model and the GEC model. In Section IV, several measures are applied to identify the order of the Markov model that satisfactorily approximates the channel, for a wide range of SNRs and fading bandwidth. Fading parameters in which the GEC model is a good approximation to the discrete communication system are identified in Section V. Concluding remarks are given in Section VI.

\section{Channel Model}

We consider a communication system that employs $M$-ary FSK modulation, a time-correlated flat Rician-fading channel, and noncoherent demodulation. The complex envelope of the received signal at the input to the demodulator is corrupted by a multiplicative Rician fading and by an additive white Gaussian noise with the one-sided power spectral density equal to $N_{0}$. The complex envelope of the fading process $\tilde{G}(t)=\tilde{G}_{I}(t)+$ $\jmath \tilde{G}_{Q}(t)$ is a complex, wide-sense stationary, Gaussian process with real constant mean $\eta$, where $\jmath=\sqrt{-1}$ and the quadrature components $\tilde{G}_{I}(t)$ and $\tilde{G}_{Q}(t)$ are mutually independent Gaussian process with the same covariance function, named $C(\tau)$. Although the analysis carried out here can be applied to a fading process with arbitrary covariance function, we adopted the Clarke's model [29], [30] for $C(\tau)$

$$
C(\tau)=\frac{1}{2} \mathbf{E}\left\{[\tilde{G}(t+\tau)-\eta]\left[\tilde{G}^{\star}(t)-\eta\right]\right\}=\sigma_{g}^{2} J_{0}\left(2 \pi f_{D} \tau\right)
$$

where $J_{0}(x)$ is the zero-order Bessel function of the first kind, $f_{D}$ is the maximum Doppler frequency, and $\sigma_{g}^{2}$ is the variance of $\tilde{G}(t)$. For a fixed time instant, the fading envelope $A_{k} \triangleq$ $\sqrt{\tilde{G}_{I}^{2}(k T)+\tilde{G}_{Q}^{2}(k T)}$ (where $T$ is the symbol interval) has the Rician pdf given by

$$
p_{A}(a)=\frac{a}{\sigma_{g}^{2}} e^{-\left(\eta^{2}+a^{2}\right) / 2 \sigma_{g}^{2}} I_{0}\left(\frac{\eta a}{\sigma_{g}^{2}}\right)
$$

where $I_{0}(x)$ is the zero-order modified Bessel function of the first kind. When the inphase process is zero-mean $(\eta=0)$, the fading envelope follows the Rayleigh pdf

$$
p_{A}(a)=\frac{a}{\sigma_{g}^{2}} e^{-\left(a^{2} / 2 \sigma_{g}^{2}\right)} .
$$

At each signaling interval of length $T$, the demodulator forms the $M$ decision variables and decides which signal was more likely to have been transmitted. We define a binary error process $\left\{E_{k}\right\}_{k=1}^{\infty}$, where $E_{k}=0$ indicates no symbol error at the $k$ th interval and $E_{k}=1$ indicates a symbol error. It can be shown that the probability of an error sequence of length $n, \mathbf{e}_{n}=$ $e_{1} e_{2} \ldots e_{n}$, may be expressed as [4]

$$
\begin{array}{r}
P\left(\mathbf{e}_{n}\right)=\sum_{l_{1}=e_{1}}^{M-1} \ldots \sum_{l_{n}=e_{n}}^{M-1}\left(\prod_{k=1}^{n} \frac{\left(\begin{array}{c}
M-1 \\
l_{k}
\end{array}\right)(-1)^{l_{k}+e_{k}}}{l_{k}+1}\right) \\
\times \frac{\exp \left\{-\frac{\Omega E_{s}}{N_{0}} K_{R} \mathbf{1}^{T} \mathbf{F}\left(\left(K_{R}+1\right) \mathbf{I}+\frac{\Omega E_{s}}{N_{0}} \overline{\mathbf{C F}}\right)^{-1} \mathbf{1}\right\}}{\operatorname{det}\left(\mathbf{I}+\frac{\Omega E_{s}}{N_{0}}\left(1+K_{R}\right)^{-1} \overline{\mathbf{C}} \mathbf{F}\right)}
\end{array}
$$

where the $(i, j)$ th entry of the normalized $n \times n$ covariance matrix $\overline{\mathbf{C}}$ is $J_{0}\left(2 \pi f_{D}(i-j) T\right), \mathbf{F}$ is a diagonal matrix defined as $\mathbf{F}=\operatorname{diag}\left(l_{1} / l_{1}+1, \ldots, l_{n} / l_{n}+1\right), E_{s}$ is the energy of the transmitted symbol, $K_{R}=\eta^{2} / 2 \sigma_{g}^{2}$ is the Rician factor, $\mathbf{1}$ is a column vector of ones, $\Omega=\eta^{2}+2 \sigma_{g}^{2}$, and the superscript $[\cdot]^{T}$ indicates the transpose of a matrix. We consider $\Omega=1$; that is, the received average energy $\left(\Omega E_{s}\right)$ is not affected by the fading. We will call this discrete fading model from the modulator input to the demodulator output the discrete channel with Clarke's autocorrelation (DCCA) model. Hereafter, we consider binary modulation $(M=2)$, so the DCCA model has three parameters: $K_{R}, f_{D} T$, and $E_{s} / N_{0}$.

Equation (3) can be used to calculate the probability of any error event relevant to the analysis of the DCCA fading model. For example, the probability the error bit is a 1 is

$$
P(1)=\frac{1+K_{R}}{2+2 K_{R}+\frac{E_{s}}{N_{0}}} e^{-\left(K_{R}\left(E_{s} / N_{0}\right) / 2+2 K_{R}+\left(E_{s} / N_{0}\right)\right)}
$$

and the probability of two consecutive ones (errors) is

$$
\begin{aligned}
P(11)= & \frac{\left(1+K_{R}\right)^{2}}{\left(2+2 K_{R}+\frac{E_{s}}{N_{0}}\right)^{2}-\left(\rho \frac{E_{s}}{N_{0}}\right)^{2}} \\
& \quad \times e^{-2\left(K_{R}\left(E_{s} / N_{0}\right) / 2+2 K_{R}+(\rho+1)\left(E_{s} / N_{0}\right)\right)}
\end{aligned}
$$

where $\rho$ is the correlation coefficient of two consecutive samples of the fading process $\tilde{G}(t)$

$$
\rho=J_{0}\left(2 \pi f_{D} T\right) .
$$

Equation (3) will be employed to parameterize an FSC model that accurately reflects the statistical description of the real error process. A brief description of FSC models is given next.

Consider $\left\{S_{k}\right\}_{k=0}^{\infty}$, an $N$-state first-order Markov chain with a finite state space $\overline{\mathcal{N}}_{N}=\{0,1, \ldots, N-1\}$. Let $\mathbf{P}$ be an $N \times N$ transition probability matrix whose $(i, j)$ th entry is the transition probability $p_{i, j}=P\left(S_{k}=j \mid S_{k-1}=i\right), i, j \in \mathcal{N}_{N}$. The FSC model generates an error symbol according to the following probabilistic mechanism. At the $k$ th time interval, the chain makes a transition from state $S_{k-1}=i$ to $S_{k}=j$ with probability $p_{i, j}$ and generates an output (error) symbol $e_{k} \in \mathcal{N}_{2}$ (independent of $i$ ) with probability $b_{j, e_{k}}=P\left(E_{k}=e_{k} \mid S_{k}=j\right)$. Conditioned on the state process, the error process is memoryless; that is, $P\left(\mathbf{e}_{n} \mid \mathbf{s}_{n}\right)=\prod_{k=1}^{n} P\left(e_{k} \mid s_{k}\right)$. We are assuming that the distribution of the initial state is the stationary distribution $\Pi=\left[\pi_{0}, \pi_{1}, \ldots, \pi_{N-1}\right]^{T}$. The probability of an error sequence generated by the FSC model, conditioned on the initial state, is

$$
\begin{aligned}
P\left(\mathbf{e}_{n} \mid s_{0}\right) & =\sum_{\mathbf{s}_{n}} P\left(\mathbf{e}_{n} \mid \mathbf{s}_{n}, s_{0}\right) P\left(\mathbf{s}_{n} \mid s_{0}\right) \\
& =\sum_{\mathbf{s}_{n}} \prod_{k=1}^{n} b_{s_{k}, e_{k}} p_{s_{k-1}, s_{k}} .
\end{aligned}
$$

Hence

$$
P\left(\mathbf{e}_{n}\right)=\sum_{s_{0}=0}^{N-1} \pi_{s_{0}} \sum_{\mathbf{s}_{n}} \prod_{k=1}^{n} b_{s_{k}, e_{k}} p_{s_{k-1}, s_{k}}
$$

which can be rewritten in the matrix form. Define two $N \times N$ matrices $\mathbf{P}(0)$ and $\mathbf{P}(1)$ where $\mathbf{P}=\mathbf{P}(0)+\mathbf{P}(1)$. The $(i, j)$ th entry of the matrix $\mathbf{P}\left(e_{k}\right), e_{k} \in\{0,1\}$ is $P\left(E_{k}=e_{k}, S_{k}=j \mid\right.$ 


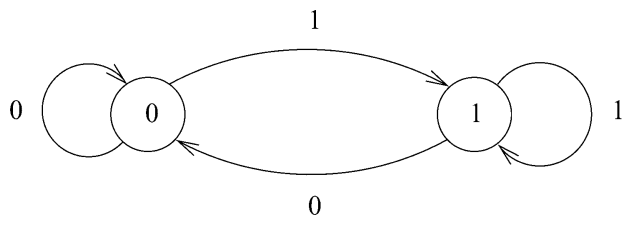

Fig. 1. First-order binary Markov model.

$\left.S_{k-1}=i\right)=b_{j, e_{k}} p_{i, j}$, which is the probability that the output symbol is $e_{k}$ when the chain makes a transition from state $i$ to $j$. Equation (7) has a matrix form given by

$$
P\left(\mathbf{e}_{n}\right)=\boldsymbol{\Pi}^{T}\left(\prod_{k=1}^{n} \mathbf{P}\left(e_{k}\right)\right) \mathbf{1} .
$$

The FSC model is completely specified by the matrices $\mathbf{P}(0)$ and $\mathbf{P}(1)$. In Section III, we define relevant properties of FSC models and discuss the evaluation of its parameters.

\section{Parameterization of Specific FSC Models}

We consider two classes of FSC models: $K$ th-order Markov models and the GEC model. Following the ideas introduced in [4], the parameters of each FSC model will be expressed as functions of the probabilities of binary sequences generated by the model. Then, we apply (3) to estimate these probabilities and to parameterize FSC models that approximate the DCCA correlated fading model.

\section{A. Kth-Order Markov Models}

A discrete stochastic process $\left\{E_{n}\right\}_{n=1}^{\infty}$ is a Markov process of $K$ th-order if it obeys the relation

$$
P\left(e_{n} \mid e_{1} e_{2} \cdots e_{n-1}\right)=P\left(e_{n} \mid e_{n-K} \cdots e_{n-1}\right) .
$$

A first-order binary Markov model is a FSC model with two states $\{0,1\}$ represented by the value of $e_{k-1}$. An error symbol is produced when the chain transitions to state 1 . Otherwise, if the chain transition is to state 0 , a correct symbol is produced. Fig. 1 illustrates the model where the labels assigned to each branch are the error symbols $e_{k}$ emitted at each state transition. Following the specification of the FSC model described in Section II, $b_{0,0}=P\left(E_{k}=0 \mid S_{k}=0\right)=1$ and $b_{1,1}=P\left(E_{k}=1 \mid S_{k}=1\right)=1$. Employing (7) for error sequences of lengths 1 and 2 , we obtain $p_{i, j}=P(i j) / P(i)$, $i, j \in\{0,1\}$. For example, $P(00)=\pi_{0} p_{0,0}^{2}+\pi_{1} p_{1,0} p_{0,0}$, and $P(0)=\pi_{0} p_{0,0}+\pi_{1} p_{1,0}$ or $P(00)=p_{0,0} P(0)$. The transition probability matrix is

$$
\mathbf{P}=\left[\begin{array}{ll}
\frac{P(00)}{P(0)} & \frac{P(01)}{P(0)} \\
\frac{P(10)}{P(1)} & \frac{P(11)}{P(1)}
\end{array}\right] .
$$

The stationary vector is $\Pi=[P(0) P(1)]^{T}$ and the matrices $\mathbf{P}(0)$ and $\mathbf{P}(1)$ for the first-order Markov model are

$$
\mathbf{P}(0)=\left[\begin{array}{cc}
\frac{P(00)}{P(0)} & 0 \\
\frac{P(10)}{P(1)} & 0
\end{array}\right] ; \quad \mathbf{P}(1)=\left[\begin{array}{cc}
0 & \frac{P(01)}{P(0)} \\
0 & \frac{P(11)}{P(1)}
\end{array}\right] .
$$

The second-order binary Markov model is specified by the conditional probabilities $P\left(e_{k} \mid e_{k-2} e_{k-1}\right)$. The second-order Markov model can be represented as a function of a first-order Markov chain [31]. The idea is to define a pair of successive

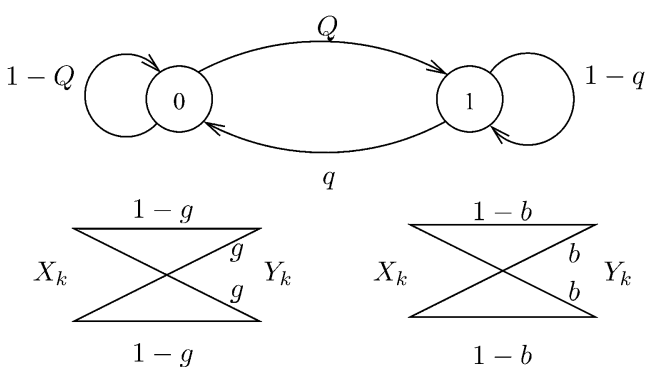

Fig. 2. Gilbert-Elliott model for burst channels.

states $e_{k-2}=i, e_{k-1}=j$ as a composite state $(i, j)$. The transition probability from the composite state $(i, j)$ to $(j, \ell)$ is

$$
P\left(e_{k}=\ell \mid e_{k-2}=i, e_{k-1}=j\right)=\frac{P(i j \ell)}{P(i j)}
$$

for $i, j, \ell \in\{0,1\}$. The transition probability from $(i, j)$ to $(h, \ell)$ is zero if $j \neq h$. The conditional probability of generating an error symbol $e_{k}$ given that the model is at the composite state $s_{k}=(j, \ell)$ is $b_{s_{k}, \ell}=1$. The methodology described above can be applied to represent $K$ th-order Markov models as a first-order Markov model. Each state of the $K$ th-order model is represented by a binary string of length $K$. Given two states $u=u_{1} u_{2} \cdots u_{K}$ and $v=v_{1} v_{2} \cdots v_{K}$, we say that $u$ and $v$ overlap progressively if $u_{2} u_{3} \cdots u_{K}=v_{1} v_{2} \cdots v_{K-1}$. If $u$ and $v$ overlap progressively, then there is a transition from $u$ to $v$ with probability $P\left(u_{1} v_{1} v_{2} \cdots v_{K}\right) / P(u)$. Otherwise, the statetransition probability is zero. Given a state $v=v_{1} v_{2} \cdots v_{K}$, $b_{v, v_{K}}=1$. For example, the matrices $\mathbf{P}(0), \mathbf{P}(1)$, and $\boldsymbol{\Pi}$ for the second-order Markov model are

$$
\begin{aligned}
\mathbf{P}(0) & =\left[\begin{array}{cccc}
\frac{P(000)}{P(00)} & 0 & 0 & 0 \\
0 & 0 & \frac{P(010)}{P(01)} & 0 \\
\frac{P(100)}{P(10)} & 0 & 0 & 0 \\
0 & 0 & \frac{P(110)}{P(11)} & 0
\end{array}\right] \\
\mathbf{P}(1) & =\left[\begin{array}{cccc}
0 & \frac{P(001)}{P(00)} & 0 & 0 \\
0 & 0 & 0 & \frac{P(011)}{P(01)} \\
0 & \frac{P(101)}{P(10)} & 0 & 0 \\
0 & 0 & 0 \frac{P(111)}{P(11)}
\end{array}\right] \\
\Pi & =\left[\begin{array}{llll}
P(00) & P(01) & P(10) & P(11)
\end{array}\right]^{T} .
\end{aligned}
$$

Clearly, the number of states grows exponentially with the order $K$.

\section{B. GEC}

The GEC is a two-state FSC model composed of state 0, which produces errors with small probability $b_{0,1} \triangleq g$ and state 1 , where errors occur with higher probability $b_{1,1} \triangleq b$, where $g \ll b$. Clearly, $b_{0,0}=1-g$ and $b_{1,0}=1-b$. The transition probabilities of the Markov chain are $p_{0,1} \triangleq Q$ and $p_{1,0} \triangleq q$, as shown in Fig. 2. The matrices $\mathbf{P}(0), \mathbf{P}(1)$, where $\mathbf{P}(0)+\mathbf{P}(1)=\mathbf{P}$, for the GEC model are given by

$$
\begin{aligned}
& \mathbf{P}(0)=\left[\begin{array}{cc}
(1-Q)(1-g) & Q(1-b) \\
q(1-g) & (1-q)(1-b)
\end{array}\right] \\
& \mathbf{P}(1)=\left[\begin{array}{cc}
(1-Q) g & Q b \\
q g & (1-q) b
\end{array}\right] .
\end{aligned}
$$


We define next the notation required in this section. Consider $\boldsymbol{\sigma}$ to be any binary sequence of finite length $Q(\sigma, i) \triangleq P\left(\mathbf{E}_{n}=\right.$ $\left.\boldsymbol{\sigma}, S_{n+1}=i\right)$. Let $\boldsymbol{\phi}$ be an empty sequence, i.e., a sequence of length zero that possesses the properties $\phi \sigma=\sigma \phi$ and $P(\phi)=$ 1 . Let $\epsilon$ and $\delta$ be binary symbols. The parameterization of the GEC is based on the following lemma. This lemma and the next proposition first appear without proof in [4]. For the sake of completeness, we offer a proof in this section.

Lemma 1: The probability of any sequence $\sigma$ generated by the GEC satisfies the following recurrence equation:

$$
P(\boldsymbol{\sigma} \epsilon \delta)=c(\epsilon, \delta) P(\boldsymbol{\sigma} \epsilon)+d(\epsilon, \delta) P(\boldsymbol{\sigma})
$$

where

$$
\begin{aligned}
c(\epsilon, \delta)= & \frac{b_{0, \epsilon}}{b_{0, \epsilon}-b_{1, \epsilon}}\left[Q b_{1, \delta}+(1-Q) b_{0, \delta}\right] \\
& -\frac{b_{1, \epsilon}}{b_{0, \epsilon}-b_{1, \epsilon}}\left[(1-q) b_{1, \delta}+q b_{0, \delta}\right] \\
d(\epsilon, \delta)= & \frac{b_{0, \epsilon} b_{1, \epsilon}}{b_{0, \epsilon}-b_{1, \epsilon}}\left[(1-q) b_{1, \delta}+q b_{0, \delta}\right] \\
& -\frac{b_{1, \epsilon} b_{0, \epsilon}}{b_{0, \epsilon}-b_{1, \epsilon}}\left[Q b_{1, \delta}+(1-Q) b_{0, \delta}\right]
\end{aligned}
$$

Proof: The probability of any sequence generated by a GEC model satisfies the relations

$$
\begin{aligned}
P(\boldsymbol{\sigma}) & =Q(\boldsymbol{\sigma}, 0)+Q(\boldsymbol{\sigma}, 1) \\
P(\boldsymbol{\sigma} \epsilon) & =Q(\boldsymbol{\sigma}, 0) b_{0, \epsilon}+Q(\boldsymbol{\sigma}, 1) b_{1, \epsilon} .
\end{aligned}
$$

Hence, $Q(\boldsymbol{\sigma}, 0)$ and $Q(\boldsymbol{\sigma}, 1)$ are expressed as

$$
\begin{aligned}
& Q(\boldsymbol{\sigma}, 0)=\frac{-b_{1, \epsilon}}{b_{0, \epsilon}-b_{1, \epsilon}} P(\boldsymbol{\sigma})+\frac{1}{b_{0, \epsilon}-b_{1, \epsilon}} P(\boldsymbol{\sigma} \epsilon) \\
& Q(\boldsymbol{\sigma}, 1)=\frac{b_{0, \epsilon}}{b_{0, \epsilon}-b_{1, \epsilon}} P(\boldsymbol{\sigma})-\frac{1}{b_{0, \epsilon}-b_{1, \epsilon}} P(\boldsymbol{\sigma} \epsilon) .
\end{aligned}
$$

The following equation also holds for the GEC model:

$$
\begin{aligned}
P(\boldsymbol{\sigma} \epsilon \delta)=Q(\boldsymbol{\sigma}, 0) b_{0, \epsilon} & {\left[Q b_{1, \delta}+(1-Q) b_{0, \delta}\right] } \\
+ & Q(\boldsymbol{\sigma}, 1) b_{1, \epsilon}\left[(1-q) b_{1, \delta}+q b_{0, \delta}\right] .
\end{aligned}
$$

Substituting (17) into (18) and rearranging the terms yields

$$
\begin{aligned}
P(\boldsymbol{\sigma} \epsilon \delta)= & \left\{\frac{b_{0, \epsilon} b_{1, \epsilon}}{b_{0, \epsilon}-b_{1, \epsilon}}\left[(1-q) b_{1, \delta}+q b_{0, \delta}\right]\right. \\
& \left.-\frac{b_{1, \epsilon} b_{0, \epsilon}}{b_{0, \epsilon}-b_{1, \epsilon}}\left[Q b_{1, \delta}+(1-Q) b_{0, \delta}\right]\right\} P(\boldsymbol{\sigma}) \\
& +\left\{\frac{b_{0, \epsilon}}{b_{0, \epsilon}-b_{1, \epsilon}}\left[Q b_{1, \delta}+(1-Q) b_{0, \delta}\right]\right. \\
& \left.\quad-\frac{b_{1, \epsilon}}{b_{0, \epsilon}-b_{1, \epsilon}}\left[(1-q) b_{1, \delta}+q b_{0, \delta}\right]\right\} P(\boldsymbol{\sigma} \epsilon) \\
= & c(\epsilon, \delta) P(\boldsymbol{\sigma} \epsilon)+d(\epsilon, \delta) P(\boldsymbol{\sigma}) .
\end{aligned}
$$

Equation (12) allows us to express $c(\epsilon, \delta)$ and $d(\epsilon, \delta)$ and, consequently, $b, g, q$, and $Q$ as functions of the probabilities of error sequences. Substituting $\boldsymbol{\sigma}=\phi$ and $\boldsymbol{\sigma}=\epsilon$ in (12) yields, respectively

$$
\begin{aligned}
P(\epsilon \delta) & =c(\epsilon, \delta) P(\epsilon)+d(\epsilon, \delta) \\
P(\epsilon \epsilon \delta) & =c(\epsilon, \delta) P(\epsilon \epsilon)+d(\epsilon, \delta) P(\epsilon) .
\end{aligned}
$$

Solving this linear system, we obtain

$$
c(\epsilon, \delta)=\frac{P(\epsilon \epsilon \delta)-P(\epsilon \delta) P(\epsilon)}{P(\epsilon \epsilon)-P^{2}(\epsilon)}
$$

and

$$
d(\epsilon, \delta)=\frac{P(\epsilon \delta) P(\epsilon \epsilon)-P(\epsilon \epsilon \delta) P(\epsilon)}{P(\epsilon \epsilon)-P^{2}(\epsilon)} .
$$

The following proposition expresses the parameters of the GEC model in terms of $c(\epsilon, \delta)$ and $d(\epsilon, \delta)$ or, consequently, in terms of the probability of error sequences of length, at most, 3 .

Proposition 1: If $P(01) \neq P(0) P(1)$, the parameters of the GEC are uniquely determined by the four probabilities: $P(0), P(00), P(000)$, and $P(111)$. The parameters $b$ and $g$ are the roots of the quadratic equation

$$
\begin{aligned}
& {[-1+c(1,1)+c(0,0)] x^{2}} \\
& \quad+[1-c(1,1)-c(0,0)+d(1,1)-d(0,0)] x-d(1,1)=0
\end{aligned}
$$

and the parameters $Q$ and $q$ are given by

$$
\begin{aligned}
Q & =\frac{c(0,0) b-c(1,1)(1-b)+(g-b)}{g-b} \\
q & =\frac{c(0,0) g-c(1,1)(1-g)+(b-g)}{b-g} .
\end{aligned}
$$

Proof: From (13) and (14), we have

$$
\begin{aligned}
& c(0,0)=(1-g)(1-Q)+(1-b)(1-q) \\
& c(1,1)=g(1-Q)+b(1-q) \\
& d(0,0)=-(1-q-Q)(1-g)(1-b) \\
& d(1,1)=-(1-q-Q) g b .
\end{aligned}
$$

From (26) and (27) and (28) and (29), respectively, we obtain

$$
\begin{aligned}
1-c(0,0)-c(1,1) & =-\mu \\
g b\left(\frac{d(0,0)}{d(1,1)}-1\right) & =1-b-g
\end{aligned}
$$

where $\mu \triangleq 1-q-Q$. Combining (29) and (31) results in the quadratic equation

$$
-\mu b^{2}+(\mu+d(0,0)-d(1,1)) b+d(1,1)=0
$$

where the same equation holds for $g$. So, substituting (30) into (32), we conclude that $b$ and $g$ are the roots of (24). Once we have determined $b$ and $g$, we use (26) and (27) to obtain (25).

\section{Model Evaluation}

This section evaluates the accuracy in which the FSC models described in Section III approximate the DCCA correlated 


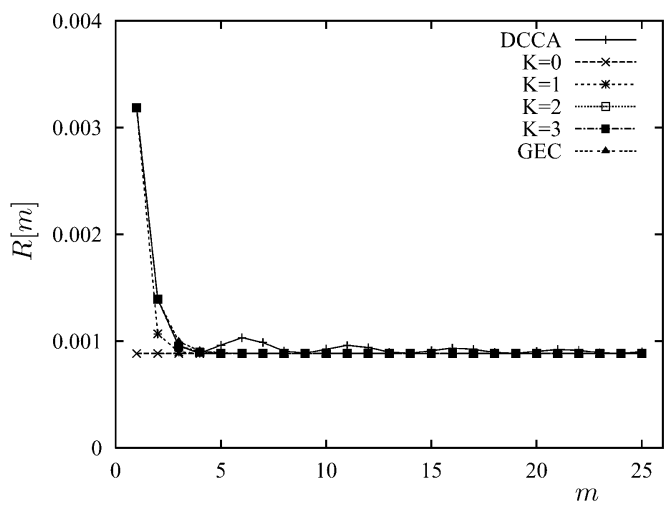

(a)

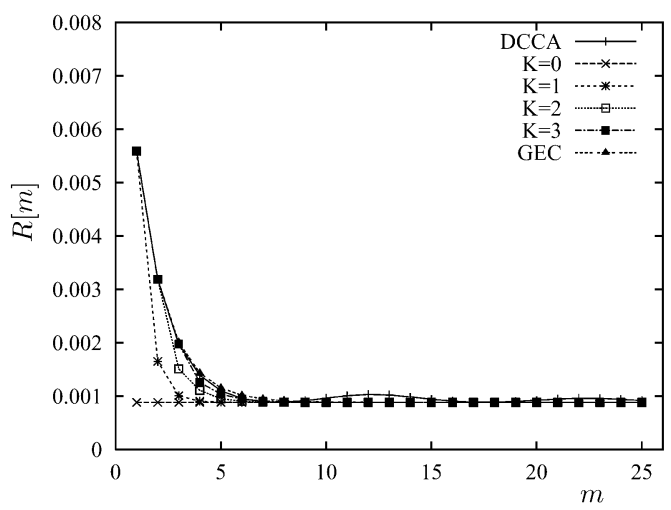

(b)

Fig. 3. Comparison of the ACFs of the DCCA fading model, the $K$ th-order Markov model $(K=0,1, \ldots, 6)$, and the GEC model. The DCCA model is Rayleigh fading $\left(K_{R}=0\right)$, with $E_{s} / N_{0}=15 \mathrm{~dB}$, and $f_{D} T=0.1$ (a), $f_{D} T=0.05$ (b).

fading model. In general, it is difficult to define a unique measure to judge if a particular model better approximates the fading channel when compared to other candidates. The criteria commonly used to make this decision include the minimization of a distance measure between the probability-of-error sequences generated by the model and by the fading channel (e.g., variational and normalized divergence), an information theoretic metric [7], and the comparison of certain statistics of the models, such as ACF and packet error rate [16].

Motivated by the results presented in [16], we next compare the ACF of the DCCA fading model with the ACF of FSC models. The ACF of a binary stationary process $\left\{E_{k}\right\}_{k=1}^{\infty}$ is given by

$$
R[m]=\mathbf{E}\left\{E_{i} E_{i+m}\right\}=P\left(E_{i}=1, E_{i+m}=1\right)
$$

where $\mathbf{E}\{X\}$ denotes the expected value of the random variable $X$. A closed-form expression for the ACF of the DCCA model is given by (5), where the correlation coefficient $\rho$ given by (6) is replaced by $\rho(m)=J_{0}\left(2 \pi m f_{D} T\right)$. Then, it follows from (5) that for the special case of Rayleigh fading $\left(K_{R}=0\right)$

$$
R[m]=\frac{1}{\left(2+\frac{E_{s}}{N_{0}}\right)^{2}-\left(\rho(m) \frac{E_{s}}{N_{0}}\right)^{2}}
$$

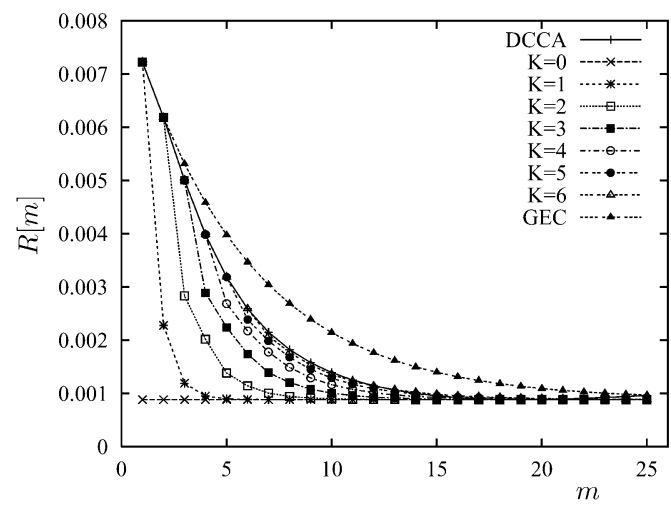

(a)

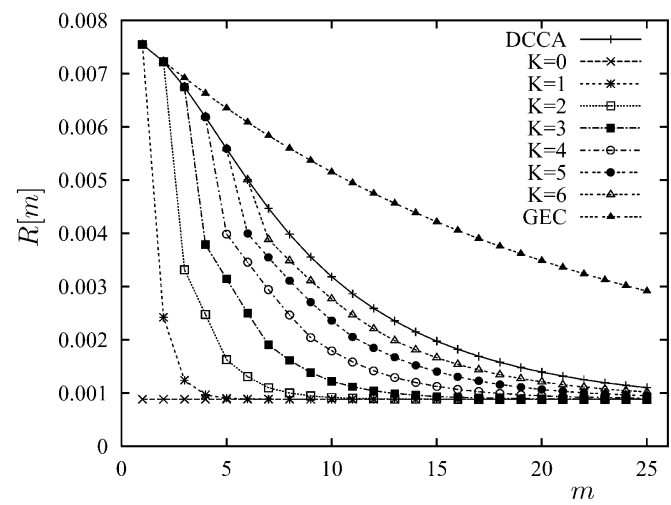

(b)

Fig. 4. Comparison of the ACFs of the DCCA fading model, the $K$ th-order Markov model $(K=0,1, \ldots, 6)$, and the GEC model. The DCCA model is Rayleigh fading $\left(K_{R}=0\right)$, with $E_{s} / N_{0}=15 \mathrm{~dB}$, and $f_{D} T=0.02$ (a), $f_{D} T=0.01(\mathrm{~b})$.

The ACF of an FSC model described by the matrices $\mathbf{P}(0)$ and $\mathbf{P}(1)$ is expressed as [23]

$$
R[m]=\Pi^{T}\left(\mathbf{P}(1) \mathbf{P}^{m-1} \mathbf{P}(1)\right) \mathbf{1}
$$

for $m \geq 1$

\section{A. Rayleigh Fading}

The ACF over 20 values of $m$ of the DCCA and the FSC models are compared in Figs. 3 and 4. The parameters of the DCCA model are $K_{R}=0, E_{s} / N_{0}=15 \mathrm{~dB}$, and $f_{D} T=0.1$, 0.05 (Fig. 3) and $f_{D} T=0.02,0.01$ (Fig. 4). Markov models of orders up to six have been considered. It is observed in Fig. 3(a) that there is a significant gain in accuracy when the order of the Markov model is increased from $K=0$ (memoryless) to $K=1$. A little gain is obtained for $K=2$ and no further gain is observed for $K=3$. Also, the ACFs of the second-order Markov and the GEC models are very alike. The curves indicate that the first-order Markov model satisfactorily approximates the DCCA fading model for $f_{D} T=0.1$. It is worth mentioning that we could have chosen either the second-order or GEC model to approximate the DCCA model, since the ACFs of these three models can barely be distinguished in Fig. 3(a). However, we want to obtain as simple an analytical model as possible with acceptable accuracy. This tradeoff between accuracy and complexity makes this decision somewhat arbitrary. When the fading rate gets slower, the order of the Markov model 


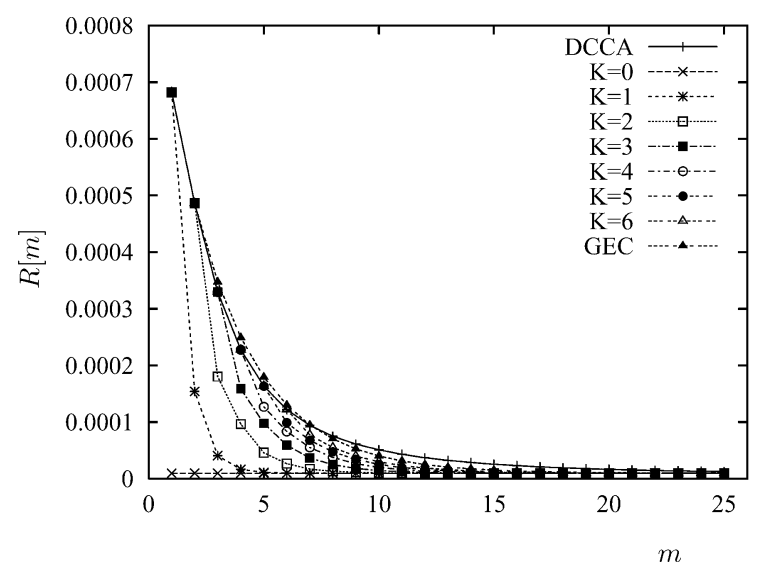

Fig. 5. Comparison of the ACFs of the DCCA fading model, the $K$ th-order Markov model $(K=0,1, \ldots, 6)$, and the GEC model. The DCCA model is Rayleigh-fading $\left(K_{R}=0\right)$ with $E_{s} / N_{0}=25 \mathrm{~dB}, f_{D} T=0.01$.

increases, as expected. For example, we observe from Fig. 3(b) that the second-order Markov is satisfactory for $f_{D} T=0.05$. However, we notice that the ACF of the third-order Markov model is a bit closer to that of the DCCA model, but this strictness may not compensate for the doubling of the number of states (we will use other statistics to confirm this assumption later). Again, the ACFs of the third-order Markov and the GEC models are very similar. When $f_{D} T \lesssim 0.04$ (curves not shown), the ACF of the GEC model diverges from the ACF of the DCCA model. This fact is illustrated in Fig. 4(a), where the curve of the fifth-order Markov model better approximates the ACF of the DCCA model than that of the GEC model. This value of $K$ can be considered as a satisfactory approximation of the DCCA model for $f_{D} T=0.02$. Fig. 4(b) indicates that the GEC model is a poor approximation and $K>6$ should be used for $f_{D} T=0.01$. Notice that the ACF of the $K$ th-order Markov model matches that of the DCCA model perfectly over an interval of length $K$.

Markov models may not be practical for very slowly fading channels $\left(f_{D} T<0.01\right)$, since the number of states grows exponentially with $K$ and large data sizes are necessary to parameterize the model. Fig. 5 displays a similar comparison for the case $E_{s} / N_{0}=25 \mathrm{~dB}, f_{D} T=0.01$. It is observed that the ACF of the DCCA model decreases more rapidly with $m$ when compared to Fig. 4(b), indicating a potential to reduce the order of the Markov approximation. It is also observed that the GEC model becomes accurate for a wider range of $f_{D} T$ when the SNR increases.

In order to verify the order of the Markov model indicated by the ACF method using a different perspective, we calculate the variational distance between the $n$-dimensional target measure $P\left(\mathbf{e}_{n}\right)$ given by (3) and the measure obtained by the $K$ th-order Markov model, namely, $P^{(K)}\left(\mathbf{e}_{n}\right)$, which is calculated using (8). The matrices $\mathbf{P}(0)$ and $\mathbf{P}(1)$ are described in Section III. The variational distance is defined as

$$
d_{v}\left(P\left(\mathbf{e}_{n}\right), P^{(K)}\left(\mathbf{e}_{n}\right)\right)=\sum_{\mathbf{e}_{n}}\left|P\left(\mathbf{e}_{n}\right)-P^{(K)}\left(\mathbf{e}_{n}\right)\right| .
$$

Fig. 6 reports the variational distance versus the order $K$ for several values of $f_{D} T$, for $E_{s} / N_{0}=15 \mathrm{~dB}$ (a) and $E_{s} / N_{0}=25 \mathrm{~dB}(\mathrm{~b})$. Because of the complexity in obtaining the $2^{n}$ possible values of $P\left(\mathbf{e}_{n}\right)$, we have considered $n=10$.

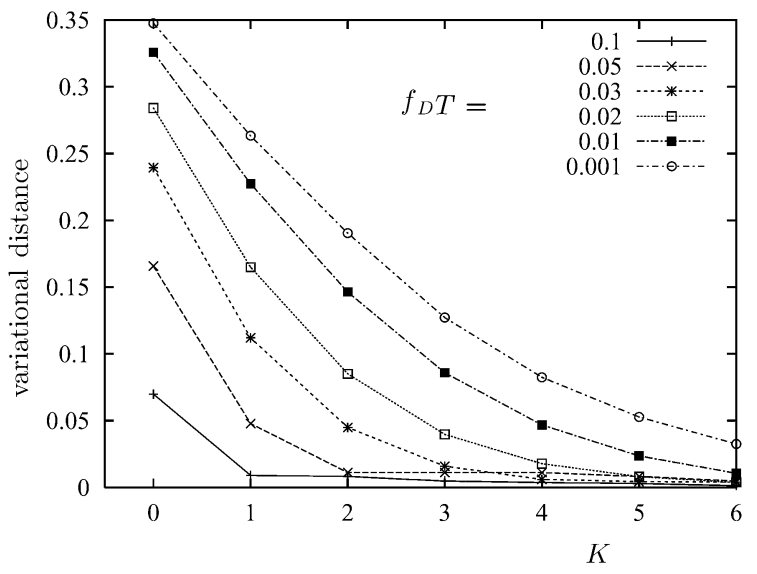

(a)

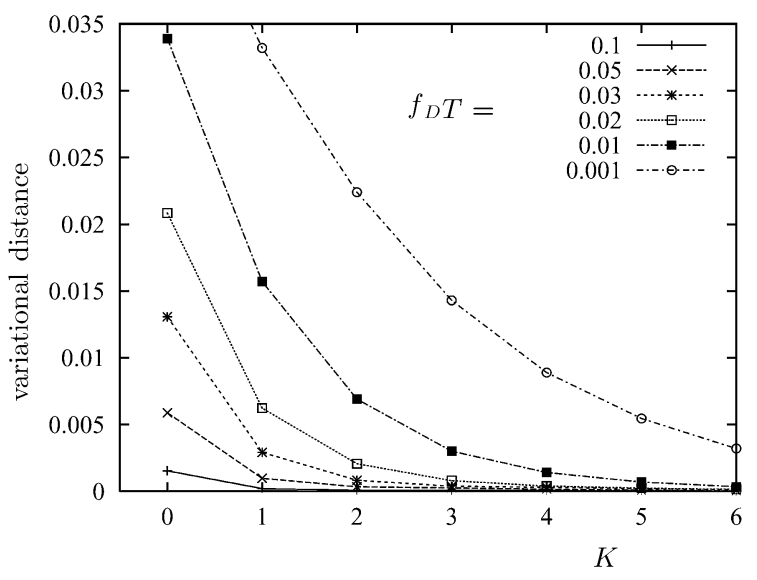

(b)

Fig. 6. Variational distance versus the order $K$ having $f_{D} T$ as a parameter. Rayleigh-fading $\left(K_{R}=0\right)$, (a) $E_{s} / N_{0}=15 \mathrm{~dB}$, (b) $E_{s} / N_{0}=25 \mathrm{~dB}$. $f_{D} T=0.1,0.05,0.03,0.02,0.01,0.001$.

TABLE I

ORDER OF THE MARKov MODEL THAT APPROXIMATES THE DCCA RAYLEIGH-FADING MODEL FOR SEVERAL VALUES OF $f_{D} T$

\begin{tabular}{c|c|c|c}
\hline$f_{D} T$ & $K_{0}\left(E_{s} / N_{0}=10 \mathrm{~dB}\right)$ & $K_{0}\left(E_{s} / N_{0}=15 \mathrm{~dB}\right)$ & $K_{0}\left(E_{s} / N_{0}=25 \mathrm{~dB}\right)$ \\
\hline 0.3 & 0 & 0 & 0 \\
0.2 & 1 & 1 & 0 \\
0.1 & 2 & 1 & 1 \\
0.05 & 3 & 2 & 2 \\
0.04 & 4 & 3 & 2 \\
0.03 & 5 & 5 & 3 \\
0.02 & $>6$ & $>6$ & 4 \\
0.01 & $>7$ & & 5 \\
\hline
\end{tabular}

A smaller distance value indicates that the $K$ th-order Markov model agrees with the DCCA model better. We say that the order of the Markov chain is $K_{0}$, when the distance converges to approximately a constant value for $K \geq K_{0}$. (The choice of a particular value of $K_{0}$, as mentioned before, is somewhat arbitrary.) The orders indicated by the convergence of the variational distance, for the range of fading environments investigated, are consistent with those obtained by the ACF method. Table I summarizes the choice of $K_{0}$ deducted from the curves for selected values of $f_{D} T$ (the curves used to 


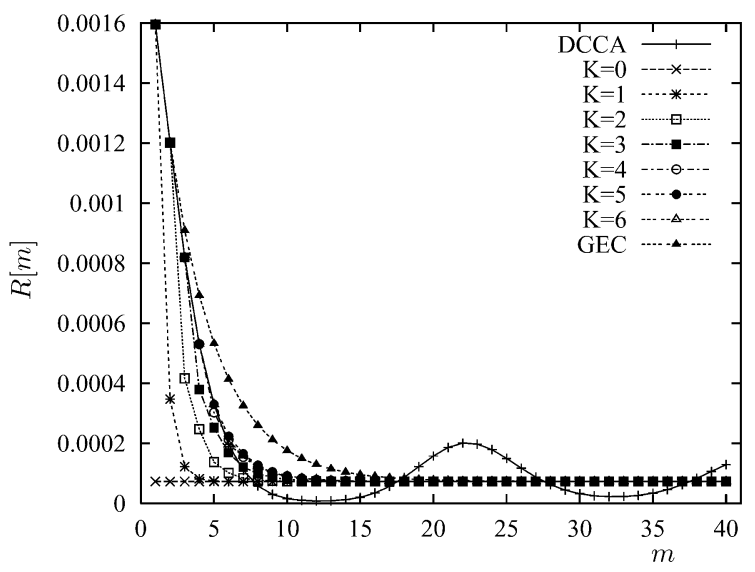

(a)

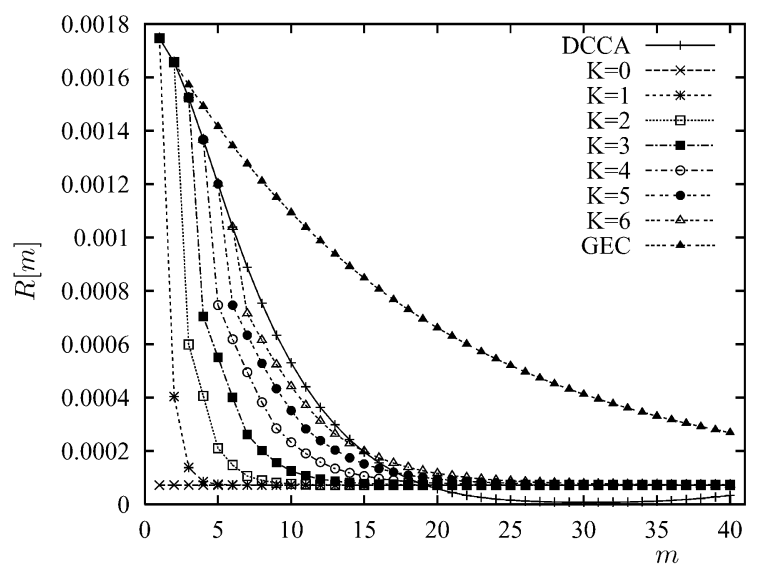

(b)

Fig. 7. Comparison of the ACFs of the DCCA fading model, the $K$ th-order Markov model $(K=0,1, \ldots, 6)$, and the GEC model. The DCCA model is Rician-fading $\left(K_{R}=5 \mathrm{~dB}\right)$ with $E_{s} / N_{0}=15 \mathrm{~dB}$, (a) $f_{D} T=0.05$, (b) $f_{D} T=0.02$.

estimate $K_{0}$ for $E_{s} / N_{0}=10 \mathrm{~dB}$ were not shown for brevity). For the range of SNR considered, a memoryless model results for fast fading $\left(f_{D} T>0.3\right)$, while a first-order Markov model is adequate for $f_{D} T=0.1$. We notice that, for moderate fading rate $\left(0.05 \leq f_{D} T \leq 0.01\right)$, the increase of the SNR from 10 to $15 \mathrm{~dB}$ and from 15 to $25 \mathrm{~dB}$ reduces the estimated order $K_{0}$ by 1 .

\section{B. Rician Fading}

The analysis presented in Section IV-A is now employed to the DCCA model with Rician fading. ACF curves are plotted in Fig. 7 for Rician fading with $K_{R}=5 \mathrm{~dB}, E_{s} / N_{0}=15 \mathrm{~dB}$, (a) $f_{D} T=0.05$, (b) $f_{D} T=0.02$. For small values of $m$ [ $m \leq 8$ in Fig. 7(a) and $m \leq 19$ in Fig. 7(b)], the ACF of the DCCA model has a monotonic decreasing exponential behavior that is well approximated by that of FSC models. However, a good fit is not possible at the oscillatory portion of the AFC curve. A comparison of Fig. 7 with Figs. 3(b) and 4(a)reveals that the Markov models provide a better fit for Rayleigh fading when $E_{s} / N_{0}=15 \mathrm{~dB}$. On the other hand, Fig. 8 shows that for $E_{s} / N_{0}=25 \mathrm{~dB}$ the differences between the ACF of the DCCA and Markov models are greatly reduced. We observe from Fig. 8(b) that the GEC model is accurate for $f_{D} T=0.02$.

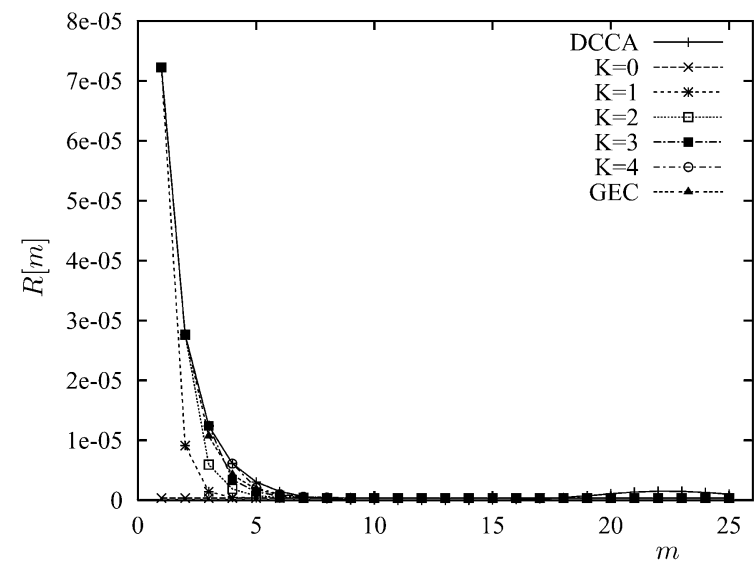

(a)

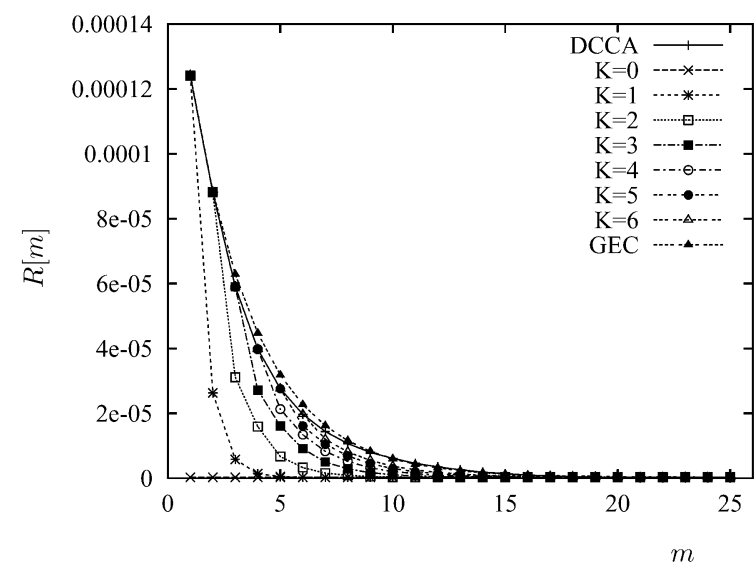

(b)

Fig. 8. Comparison of the ACFs of the DCCA fading model, the $K$ th-order Markov model $(K=0,1, \ldots, 6)$, and the GEC model. The DCCA model is Rician-fading $\left(K_{R}=5 \mathrm{~dB}\right)$ with $E_{s} / N_{0}=25 \mathrm{~dB}$, (a) $f_{D} T=0.05$, (b) $f_{D} T=0.02$.

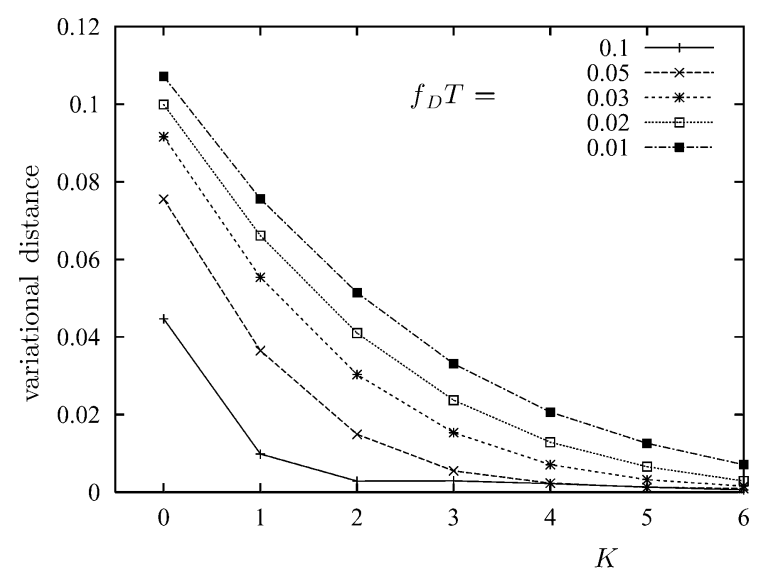

Fig. 9. Variational distance versus the order $K$ having $f_{D} T$ as a parameter Rician-fading $\left(K_{R}=5 \mathrm{~dB}\right), E_{s} / N_{0}=15 \mathrm{~dB}, f_{D} T=0.1,0.05,0.03,0.02$, 0.01

The variational distance versus the order of the Markov model $K$ is presented in Fig. 9 for several values of $f_{D} T$, block length $n=10, K_{R}=5 \mathrm{~dB}, E_{s} / N_{0}=15 \mathrm{~dB}$. The estimated orders of the Markov model $K_{0}$ obtained from the ACF curves and the convergence of the variational distance are shown in Table II. The values of $K_{0}$ in Table II are greater than their corresponding 
TABLE II

ORDER OF THE MARKOV MODEL THAT APPROXIMATES THE DCCA Rician-FAding ChanNel For SEVERAL VAlues of $f_{D} T . K_{R}=5 \mathrm{~dB}$

\begin{tabular}{c|c|c|c}
\hline$f_{D} T$ & $K_{0}\left(E_{s} / N_{0}=10 \mathrm{~dB}\right)$ & $K_{0}\left(E_{s} / N_{0}=15 \mathrm{~dB}\right)$ & $K_{0}\left(E_{s} / N_{0}=25 \mathrm{~dB}\right)$ \\
\hline 0.1 & 3 & 2 & 2 \\
0.05 & 5 & 4 & 2 \\
0.04 & 6 & 5 & 3 \\
0.03 & $>6$ & 6 & 4 \\
0.02 & $>6$ & $>6$ & 5 \\
0.01 & $>7$ & $>6$ & $>6$ \\
\hline
\end{tabular}

TABLE III

Order of THE MARKov Model That ApProximates THE DCCA Rician-Fading Channel for SeVERAL VAlues of $f_{D} T . E_{s} / N_{0}=15 \mathrm{~dB}$

\begin{tabular}{c|c|c}
\hline$f_{D} T$ & $K_{0}\left(K_{R}=2 \mathrm{~dB}\right)$ & $K_{0}\left(K_{R}=8 \mathrm{~dB}\right)$ \\
\hline 0.1 & 3 & 3 \\
0.05 & 4 & 5 \\
0.04 & 5 & 6 \\
0.03 & 6 & $>6$ \\
0.02 & $>6$ & $>6$ \\
0.01 & $>7$ & $>7$ \\
\hline
\end{tabular}

TABLE IV

RANGE OF FADING PARAMETERS WHERE THE GEC MODEL IS EQUiVALENT TO THE DCCA MODEL

\begin{tabular}{c|c|c|c}
\hline$E_{s} / N_{0}$ & Rayleigh & Rice $\left(K_{R}=2 \mathrm{~dB}\right)$ & Rice $\left(K_{R}=5 \mathrm{~dB}\right)$ \\
\hline $15 \mathrm{~dB}$ & $0.05 \leq f_{D} T<0.1$ & $0.07 \leq f_{D} T<0.1$ & - \\
$25 \mathrm{~dB}$ & $0.01 \leq f_{D} T<0.1$ & $0.01 \leq f_{D} T<0.1$ & $0.02 \leq f_{D} T<0.1$ \\
\hline
\end{tabular}

values in Table I. This can be explained by the fact that the mismatch of the ACF curves for Rician fading reflects in the convergence rate of the variational distance. It is worth mentioning that the Markov models indicated in Table II for $E_{s} / N_{0} \leq 15 \mathrm{~dB}$ reach good approximation at the first portion of the ACF curve (small $m$ ).

We have investigated the effect of the Rician factor on the estimated order $K_{0}$. We have repeated the analysis for $K_{R}=$ $2 \mathrm{~dB}$ and $K_{R}=8 \mathrm{~dB}$ and have found model orders similar to those given in Table II. Specific models are shown in Table III for $E_{s} / N_{0}=15 \mathrm{~dB}, K_{R}=2 \mathrm{~dB}$, and $K_{R}=8 \mathrm{~dB}$. The curves of several statistics show that the order $K_{0}$ is relatively insensitive to a small variation of the Rician factor. We may consider that, for the range of values considered, the increase of the Rician factor by $3 \mathrm{~dB}$ increases the estimated order by 1 .

\section{EQuivalence Between DCCA AND GEC Models}

Tables I and II indicate that the number of states of the Markov models may grow to an inconvenient size for slow fading. We have also observed that the more compact two-state GEC and DCCA models exhibit greater ACF discrepancies when $f_{D} T$ becomes smaller. It is, therefore, of interest to

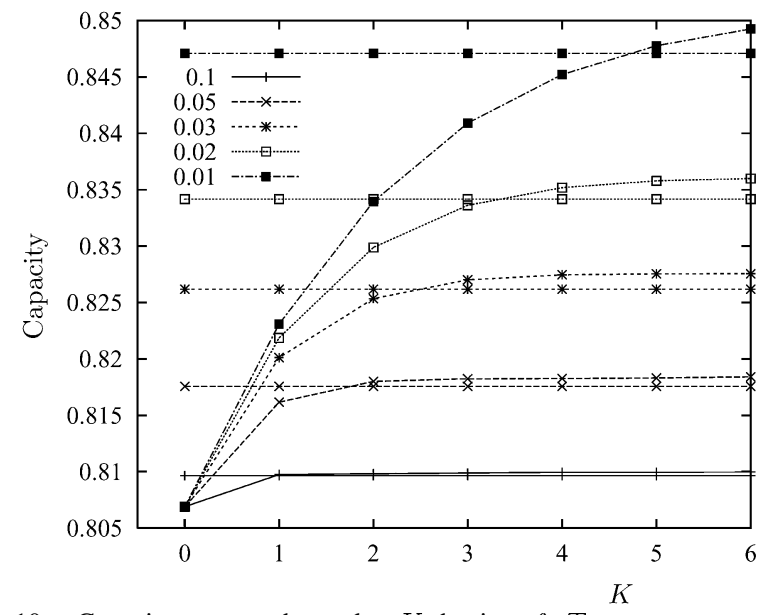

Fig. 10. Capacity versus the order $K$ having $f_{D} T$ as a parameter for $E_{s} / N_{0}=15 \mathrm{~dB}$. Rayleigh-fading $\left(K_{R}=0\right), f_{D} T=0.1,0.05,0.03,0.02$, 0.01 .

evaluate the effectiveness of the GEC model for a wide range of fading parameters. Table IV classifies the minimum value of $f_{D} T$, in which the GEC model is approximately statistically equivalent to the DCCA model. Figs. 3(b), 5(b), and 8(b) verify the accuracy of the GEC model at the lower bound to $f_{D} T$ shown in Table IV. This table shows that, for Rayleigh fading, $E_{s} / N_{0}=25 \mathrm{~dB}, f_{D} T=0.01$, the GEC model may be an interesting alternative with respect to the 32-state Markov model indicated in Table I. We found that the GEC model is not adequate when $E_{s} / N_{0}<15 \mathrm{~dB}$. Although the GEC model is suitable for fast fading, the zeroth- and first-order Markov models are simpler to analyze. This is the reason for the upper bound $f_{D} T=0.1$ in Table IV. For a given SNR, the range of fading rates where the GEC model is accurate becomes narrower when the Rician factor increases.

To investigate this equivalence further, we will compare the capacity of FSC models. The capacity of the Markov models has closed-form solution, while the capacity of the GEC model was calculated using the algorithm published by Mushkin and Bar-David [32]. In Fig. 10, the capacities are plotted versus the Markov order $K$, for $E_{s} / N_{0}=15 \mathrm{~dB}, K_{R}=0$. The flat curves correspond to the capacities of the GEC models. For each $f_{D} T$, the capacity of the Markov models increases with $K$ and converges to the capacity of the DCCA model. The estimated values of $K_{0}$ indicated by the convergence of the capacity curves agree with those shown in Tables I and II. For each $f_{D} T$, a crossover between the capacity curves reveals the value of $K$ where the $K$ th-order Markov model and the GEC model have similar capacities. If this value of $K$ agrees with the value indicated in Table I, we have established an equivalence between the GEC and DCCA models.

Our results indicate that, for practical values of SNR, the GEC model is valid for moderate fading rates. ACF curves for very slow Rayleigh-fading $\left(f_{D} T=0.001\right)$ are shown in Fig. 11. It is seen that Markov models are infeasible and that more sophisticated hidden Markov Models are required.

\section{A. Codeword Error Probability (PCE)}

In order to investigate the impact of the results shown in Tables I, II, and IV on higher layer protocols, we discuss the effect 


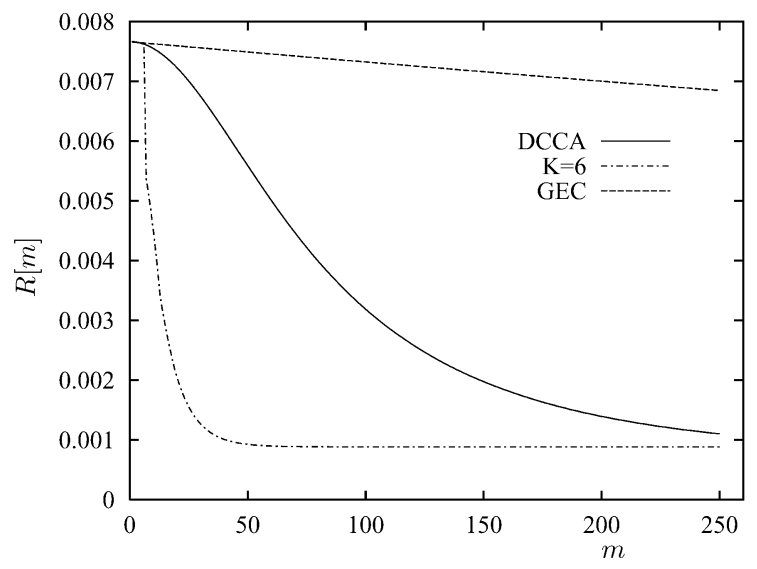

Fig. 11. Comparison of the ACFs of the DCCA fading model, the sixth-order Markov model, and the GEC model. The DCCA model is Rayleigh-fading $\left(K_{R}=0\right)$ with $E_{s} / N_{0}=15 \mathrm{~dB}, f_{D} T=0.001$.

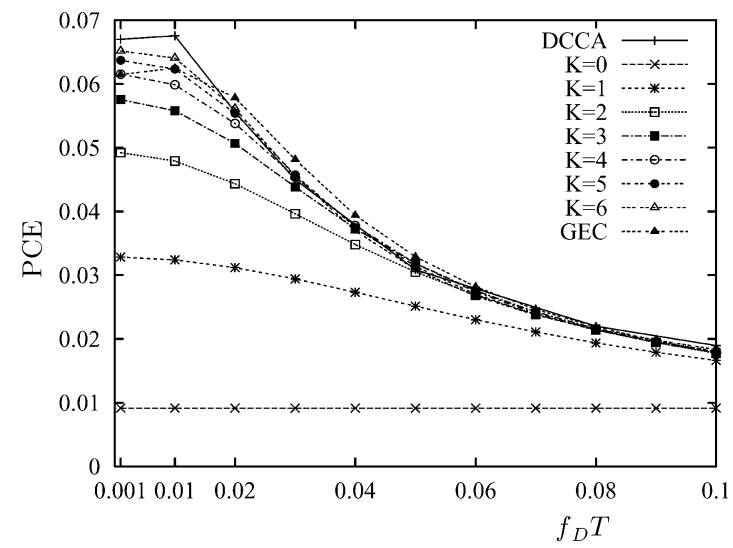

(a)

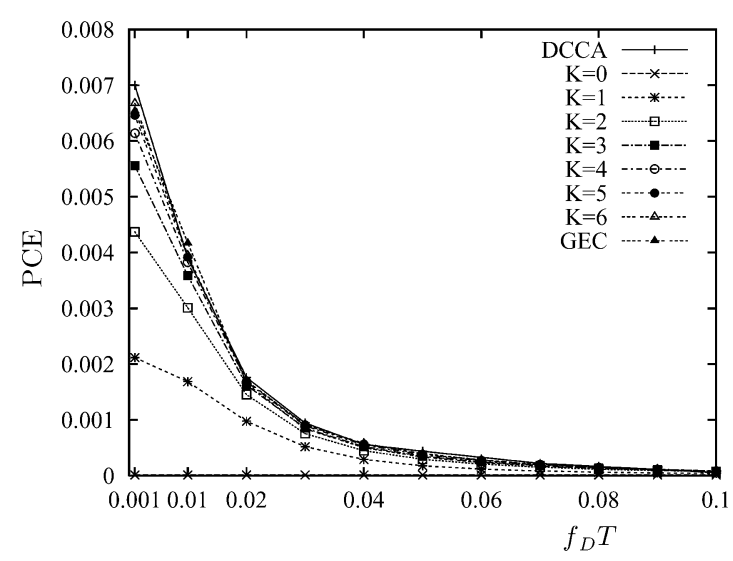

(b)

Fig. 12. Codeword error probability (PCE) versus $f_{D} T$ of the $K$ th-order Markov model $(K=0,1, \ldots, 6)$, the GEC model, and the DCCA fading model (simulations). The DCCA model is Rayleigh fading with (a) $E_{s} / N_{0}=15 \mathrm{~dB}$ and (b) $E_{s} / N_{0}=25 \mathrm{~dB}$

of the channel models on the PCE of block codes. We consider the binary $(15,7)$ block code [33] with error-correcting capability $t=2$. The PCE is defined as the probability of occurrence of received words with more than $t$ erroneous symbols. Thus

$$
\mathrm{PCE}=1-\sum_{m=0}^{t} P(m, n)=\sum_{m=t+1}^{n} P(m, n)
$$

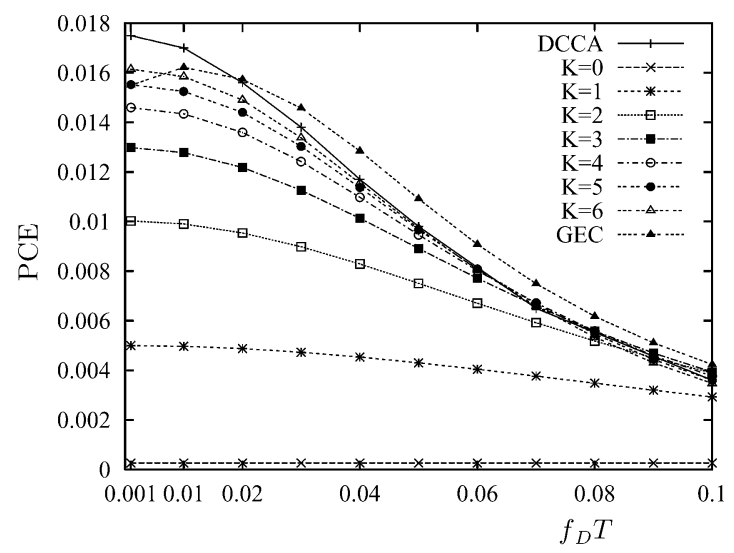

(a)

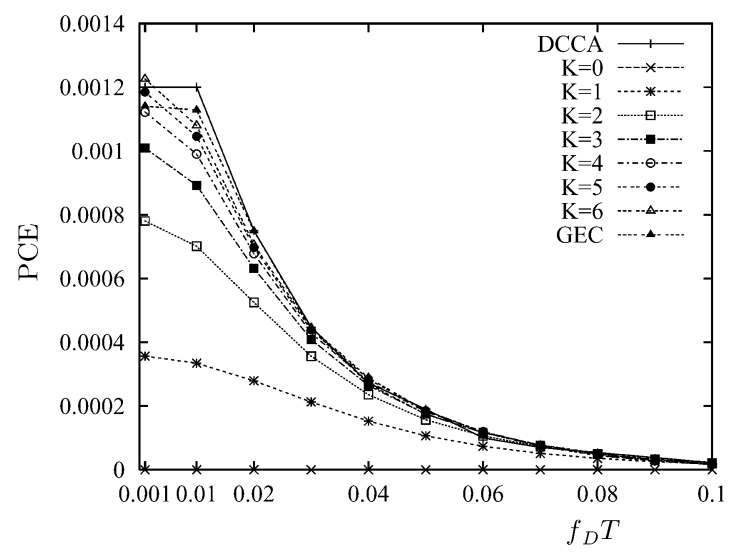

(b)

Fig. 13. PCE versus $f_{D} T$ of the $K$ th-order Markov model $(K=$ $0,1, \ldots, 6)$, the GEC model, and the DCCA fading model (simulations). The DCCA model is Rician fading $\left(K_{R}=5 \mathrm{~dB}\right)$ with (a) $E_{s} / N_{0}=15 \mathrm{~dB}$ and (b) $E_{s} / N_{0}=25 \mathrm{~dB}$.

where $P(m, n)$ is the probability the FSC model generates $m$ errors in a block of length $n$.

Fig. 12 presents PCE curves for FSC models as a function of $f_{D} T$, for Rayleigh fading with $E_{s} / N_{0}=$ (a) $15 \mathrm{~dB}$ and (b) $25 \mathrm{~dB}$. Simulated curves for the DCCA model are shown for comparison. We observe that the orders of the Markov models shown in Table I match the simulation results closely. The GEC model provides an excellent prediction of the performance in the range shown in Table IV. Similar conclusions can be drawn from the PCE curves for Rician fading shown in Fig. 13.

Fig. 12(b) reveals that the range of fading rates in Table IV can be expanded with no severe penalty in accuracy. For example, for $E_{s} / N_{0}=15 \mathrm{~dB}$, the GEC model may be a good approximation to the DCCA Rayleigh fading for $0.02 \leq f_{D} T<0.1$. This means that a small discrepancy on the ACF curves [compare the DCCA and GEC curves in Fig. 4(a)] may be acceptable for protocol evaluation.

\section{CONCLUSION}

We have developed FSC models that characterize the error sequence of a communication system operating over a fading channel. Markov models of order up to six have been proposed as an approximation to the DCCA model for a broad range of fading environments. We have used several criteria to estimate 
the order of the Markov process, e.g., ACF, variational distance, and capacity. These criteria lead to a similar conclusion that the $K$ th-order Markov model is a good approximation to the DCCA model. This analysis reinforces the results in [16] regarding the effectiveness of the ACF criterion to judge the fitness of FSC models to approximate correlated fading channels. The use of other criteria helps to resolve the tradeoff between accuracy and complexity.

It is observed that the first-order approximation is satisfactory to model DCCA channels with Rayleigh fading for values of $f_{D} T$ around 0.1 . In the range of SNR considered, the $K$ th-order Markov (for judiciously selected $K$ ) is an accurate model for fast and medium fading rates $\left(f_{D} T>0.02\right)$. For slower fading rates, $\left(f_{D} T<0.02\right)$ Markov models of order greater than six are required. The GEC model is not adequate for low SNR $\left(E_{s} / N_{0}<15 \mathrm{~dB}\right)$, but its becomes accurate for a broad range of fading rates when the SNR increases. Higher order models are needed to approximate Rician fading with respect to Rayleigh fading with the same fading parameters.

\section{REFERENCES}

[1] R. K. Mallik, "On multivariate Rayleigh and exponential distributions," IEEE Trans. Inform. Theory, vol. 48, pp. 1499-1515, June 2003.

[2] G. K. Karagiannidis, D. A. Zogas, and S. A. Kotsopoulos, "On the multivariate Nakagami-m distribution with exponential correlation," IEEE Trans. Commun., vol. 51, pp. 1240-1244, Aug. 2003.

[3] — _ "An efficient approach to multivariate Nakagami-m distribution using Greens's matrix approximation," IEEE Trans. Wireless Commun., vol. 2, pp. 883-889, Sept. 2003.

[4] C. Pimentel and I. F. Blake, "Modeling burst channels using partitioned Fritchman's Markov models," IEEE Trans. Veh. Technol., vol. 47, pp. 885-899, Aug. 1998.

[5] F. Swarts and H. C. Ferreira, "Markov characterization of digital fading mobile VHF channels," IEEE Trans. Veh. Technol., vol. 43, pp. 977-985, Nov. 1994.

[6] H. Wang and N. Moayeri, "Finite-state Markov channel-A useful model for radio communication channels," IEEE Trans. Veh. Technol., vol. 44, pp. 163-171, Feb. 1995.

[7] H. Wang and P. Chang, "On verifying the first-order Markovian assumption for a Rayleigh fading channel model," IEEE Trans. Veh. Technol., vol. 45, pp. 353-357, May 1996.

[8] F. Swarts and H. C. Ferreira, "Markov characterization of channels with soft decision outputs," IEEE Trans. Commun., vol. 41, pp. 678-682, May 1993.

[9] H. Bischl and E. Lutz, "Packet error rate in the noninterleaved Rayleigh channel," IEEE Trans. Commun., vol. 43, pp. 1375-1382, Feb./Mar./Apr. 1995.

[10] M. Zorzi, R. Rao, and L. B. Milstein, "ARQ error control for fading mobile radio channels," IEEE Trans. Veh. Technol., vol. 46, pp. 445-455, May 1997.

[11] Q. Zhang and S. Kassam, "Finite-state Markov model for Rayleigh fading channels," IEEE Trans. Commun., vol. 47, pp. 1688-1692, Nov. 1999.

[12] F. Babich and G. Lombardi, "A Markov model for the mobile propagation channel," IEEE Trans. Veh. Technol., vol. 49, pp. 63-73, Jan. 2000.

[13] M. Chu and W. Stark, "Effect of mobile velocity on communications in Fading channels," IEEE Trans. Veh. Technol., vol. 49, pp. 202-210, Jan. 2000.

[14] C.-D. Iskander and P. T. Mathiopoulos, "Finite-state Markov modeling of diversity Nakagami channels," presented at the Canadian Workshop Information Theory, Vancouver, BC, Canada, June 2001.
[15] J. Lu, K. B. Letaief, and M. L. Liou, "Robust video transmission over correlated mobile fading channels," IEEE Trans. Circuits Syst. Video Technol., vol. 9, pp. 737-751, Aug. 1999.

[16] C. Tan and N. C. Beaulieu, "On first-order Markov modeling for the Rayleigh fading channel," IEEE Trans. Commun., vol. 48, pp. 2032-2040, Dec. 2000.

[17] F. Babich, O. Kelly, and G. Lombardi, "Generalized Markov modeling for flat fading," IEEE Trans. Commun., vol. 48, pp. 547-551, Apr. 2000.

[18] L. Wilhelmsson and L. B. Milstein, "On the effect of imperfect interleaving for the Gilbert-Elliott channel," IEEE Trans. Commun., vol. 47, pp. 681-688, May 1999.

[19] P. Bergamo, D. Maniezzo, A. Giovanardi, G. Mazzini, and M. Zorzi, "Improved Markov model for Rayleigh fading envelop," Electron. Lett., vol. 38 , no. $10,2002$.

[20] H. Turin and R. van Nobelen, "Hidden Markov modeling of flat fading channels," IEEE J. Select. Areas Commun., vol. 16, pp. 1809-1817, Dec. 1998.

[21] M. Zorzi, "Some results on error control for burst-error channels under delay constraints," IEEE Trans. Veh. Technol., vol. 50, pp. 12-24, Jan. 2001.

[22] - "Outage and error events in burst channels," IEEE Trans. Veh. Technol., vol. 46, pp. 349-356, Mar. 2001.

[23] C. Pimentel and I. F. Blake, "Enumeration of Markov chains and burst error statistics for finite state models," IEEE Trans. Veh. Technol., vol. 48, pp. 415-428, Mar. 1999.

[24] - "Concatenated coding performance for FSK modulation on timecorrelated rician fading channels," IEEE Trans. Commun., vol. 46, pp. 1610-1618, Dec. 1998.

[25] K. Sakakibara, "Performance analysis of the error-forecasting decoding for interleaved block codes on Gilbert-Elliott channels," IEEE Trans. Commun., vol. 48, pp. 386-395, Mar. 2000.

[26] C. Pimentel, "Generating series and performance bounds for convolutional codes over burst error channels," IEEE Trans. Veh. Technol., vol. 51, pp. 1011-1017, Sept. 2002.

[27] G. Sharma, A. Hassan, and A. Dholakia, "Performance evaluation of burst-error correcting codes on a Gilbert-Elliott channel," IEEE Trans. Commun., vol. 46, pp. 846-849, July 1998.

[28] L. Zhong, F. Alajaji, and G. Takahara, "A comparative study of burstnoise communication channel models," presented at the 21st Biennial Symp. Communications, Kingston, ON, Canada, June 2002.

[29] R. H. Clarke, "A statistical theory of mobile-radio reception," Bell Syst. Tech. J., vol. 47, pp. 957-1000, 1968.

[30] M. Gans, "A power-spectral theory of propagation in the mobile radio environment," IEEE Trans. Veh. Technol., vol. VT-21, pp. 27-38, Feb. 1972.

[31] T. W. Anderson and L. A. Goodman, "Statistical inference about Markov chains," Ann. Math. Stat., vol. 28, pp. 89-110, 1957.

[32] M. Mushkin and I. Bar-David, "Capacity and coding for the Gilbert-Elliott channels," IEEE Trans. Inform. Theory, vol. 35, pp. 1277-1289, Nov. 1989.

[33] S. B. Wicker, Error Control Systems for Digital Communication and Storage. Englewood Cliffs, NJ: Prentice-Hall, 1995.

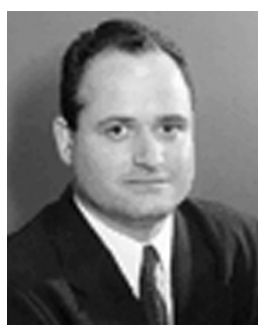

Cecilio Pimentel (S'93-M'96) was born in Recife, Brazil, in 1966. He received the B.S.E.E. degree from the Federal University of Pernambuco, Recife, Brazil, in 1987, the M.S.E.E. degree from the Catholics University of Rio de Janeiro, Rio de Janeiro, Brazil, in 1990, and the Ph.D. degree in electrical engineering from the University of Waterloo, Waterloo, ON, Canada, in 1996.

Since October 1996, he has been with the Department of Electronics and Systems, Federal University of Pernambuco, where he currently is an Associate Professor. His research interests include digital communications, information theory, and error correcting coding.

Dr. Pimentel is a Member of the Brazilian Telecommunication Society (SBrT). 


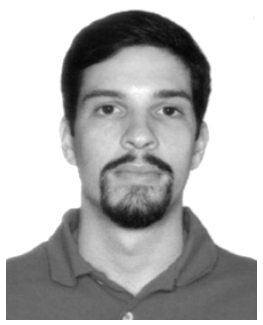

Tiago H. Falk (S'00) was born in Recife, Brazil, in September 1979. He received the B.S. degree in electrical engineering from the Federal University of Pernambuco, Recife, in 2002 and is currently working toward the M.Sc. (Eng.) degree at Queen's University, Kingston, ON, Canada.

His research interests include communication theory, channel modeling, and speech and audio processing.

Mr. Falk is a Student Member of the Brazilian Telecommunication Society (SBrT). He is the recipient of the Brazilian Concil for Scientific and Technological Development (CNPq) undergraduate scholarship and was awarded the Prof. Newton Maia Young Scientist Prize Federal University of Pernambuco (UFPE)-CNPq in 2001.

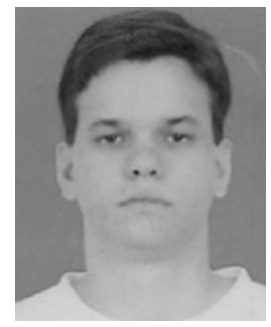

Luciano Lisbôa was born in Recife, Brazil, in September 1978. He received the B.S. degree in electrical engineering from the Federal University of Pernambuco, Recife, in 2002.

He is currently with CHESF (a hydroelectric company in Brazil), conducting research in the areas of automation, control, and protection of power electrical systems. His research interests include communication theory, channel modeling, and supervisory control and data acquisition (SCADA) Systems.

Telecommunication Society (SBrT).
Mr. Lisbôa is a Student Member of the Brazilian 